\title{
A New Region of Interest Image Coding for Narrowband Network: Partial Bitplane Alternating Shift
}

\author{
Li-Bao Zhang \\ College of Information Science and Technology of Beijing Normal University, \\ Beijing 100875, China \\ Libaozhang@163.com
}

\begin{abstract}
Regions Of Interest (ROI) image coding is one of the most significant features in JPEG2000. It allows ROIs of the image are of higher importance than background (BG). In this paper, a new and efficient scaling-based method so-called Partial Bitplane Alternating Shift (PBAShift) is described. The new algorithm firstly shifts up partial most significant bitplanes of ROI. Then, the most significant bitplanes of BG coefficients and general significant bitplanes of ROI coefficients are shifted up by bitplanes alternating scaling method. The least significant bitplanes of ROI and BG coefficients are obtained in the original position. The PBAShift method, in addition to supporting efficient single ROI coding, can flexibly code multiple ROIs with degrees of interest in an image. The experiments on remote sensing images show the presented method supports ROI coding of both arbitrary shape and arbitrary scaling without shape coding. Additionally, it can handle complexity multiple ROIs of arbitrary shapes efficiently.
\end{abstract}

\section{Introduction}

The functionality of ROI is important in applications where certain parts of the image are of higher importance than others. In such a case, these ROIs need to be encoded at higher quality than the background. During the transmission of the image, these regions need to be transmitted first or at a higher priority, as for example in the case of progressive transmission. JPEG 2000 standard in [1] and [2] not only supports ROI coding firstly, but defines two coding algorithms that are called Maxshift (maximum shift) method in part 1 and the general scaling-based method in part 2 along with the syntax of a compressed codestream. In these methods, a region of interest of the image can have a better quality than the rest at any decoding bit-rate. In other words, this implies a non-uniform distribution of the quality inside the image.

Although the Maxshift method is simple and efficient, two disadvantages are inevitable. First, this method requires decoding of all ROI coefficients before accessing bit-planes of the background and uses large shifting values that significantly increase the number of total bit-planes to encode. Second, it is difficult that this method handles multiple ROIs of any shapes.

In this paper, we present a new ROI coding scheme called PBAShift that not only retains advantages, but also alleviates the drawbacks of both ROI coding methods in 
JPEG2000. Three main strategies are used to improving the ROI coding efficiency. Firstly, the presented method shifts up partial most significant bitplanes of ROI, which can ensure that the most important bitplanes of ROI coefficients are coded and transmitted. Secondly, the most significant bitplanes of BG coefficients and general significant bitplanes of ROI coefficients are shifted up by bitplanes alternating scaling method, which enables the flexible adjustment of compression quality in ROI and BG. Finally, the least significant bitplanes of ROI and BG coefficients are obtained in the original position.

The new method is based on the embedded block coding with optimized truncation (EBCOT) scheme. It reduces the priority of the less important region or background of an image, allowing the user to quickly view the ROI with higher quality without receiving the entire image. Thus, it substantially saves the transmission time, storage space, and computational cost of image compression. Simulation results show that the new method can combine the advantages of the two standard methods of JPEG2000 and efficiently compress multiple ROIs according to different degrees of interest without any shape information.

\section{The ROI Coding in JPEG2000}

In the part one and part two of JPEG2000, two kinds of ROI coding methods are included in the standard: the Maxshift method and the general scaling based method. As illustrated in Fig. 1(b) and Fig. 1(c), these two methods place ROI associated bits in the higher bitplanes by downshifting the bits of BG coefficients from Most Significant Bitplane (MSB) to Least Significant Bitplane (LSB) [3], [4], so that ROI coefficients can be coded firstly in the embedded bitplane coding [4], [5]. In figure 1(a), no ROI code and no scales. In figure 1(b), the general scaling-based method is shows and the scaling value is 5 . In figure 1(c), the Maxshift method is shows and the scaling value is 10 .

The general scaling-based method has two major drawbacks. First, it needs to encode and transmit the shape information of the ROIs. This rapidly increases the complexity of encoder and decoder implementations. Second, if arbitrary ROI sharps are desired, then shape coding will consume a large number of bits, which significantly decreases the overall coding efficiency. To solve above problems, a new effective solution-Maxshift method was proposed for JPEG 2000. The Maxshift method is a particular case of the general scaling-based method when the scaling value is so large that there is no overlapping between BG and ROI bitplanes, i.e., so the scaling value, s, must satisfy (1):

$$
s \geq \max \left(M_{b}\right)
$$

Where $M_{b}$ is the nominal maximum number of magnitude bitplanes in subband $b$. Fig. 1(c) shows the bitplane shift in Maxshift method. All significant bits associated with the ROI after scaling will be in higher bitplanes than all the significant bits associated with the background. Therefore, ROI shape is implicit for the decoder in this method, and arbitrarily shaped ROI coding can be supported. 


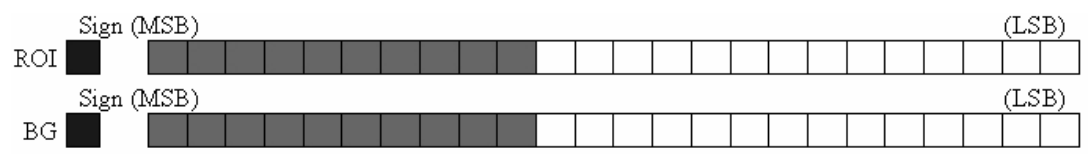

(a) No Scaling

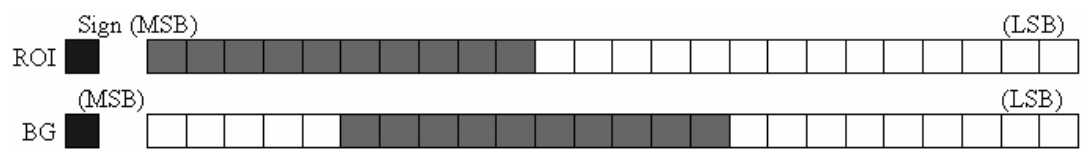

(b) General scaling based method

Sign (MSB)
ROI \begin{tabular}{|l|l|l|l|l|l|l|l|l|l|l|l|l|l|l|}
\hline \\
\hline
\end{tabular}
(MSB)

(c) Maxshift method

Fig. 1. Two basic Scaling method of ROI in JPEG2000

\section{Disadvantages of the ROI Coding in JPEG2000}

Although the Maxshift method is simple, three limitations of this method are inevitable. First, it does not have the flexibility for an arbitrary scaling value to define the relative importance of the ROI and the BG wavelet coefficients as in the general scaling-based method. Second, this method requires decoding of all ROI coefficients before accessing bit-planes of the background and uses large shifting values that significantly increase the number of total bit-planes to encode. Finally, when there are multiple ROIs in the same image, any ROI cannot have its own scaling value, and therefore different priority during encoding and transmission of the image.

Because of the limitations of two standard ROI coding algorithms, some improved methods for ROI coding were proposed. A new method was proposed in [4] with low scaling values to take advantages of two standard methods. It is implemented by removing all the overlapping bitplanes between ROI and BG coefficients, which relatively modified the quantization steps of coefficients. However, the method brought the reduction of final ROI and BG qualities. A bitplane-by-bitplane shift (BbBShift) method was proposed in [6] by shifting the bitplanes on a bitplane-by-bitplane basis instead of shifting them all at once in Maxshift method. Although it supports arbitrarily shaped ROI coding without coding shapes, it is difficult for the BbBShift method to code multiple ROIs with different priority during encoding and transmission. The partial significant bitplanes shift (PSBShift) method proposed by [7] shifts part of the most significant of ROI coefficients instead of shifting the whole bitplanes as the standard methods do. But the PSBShift method needs the same scaling values for every ROI for multiple ROIs coding. Additionally, this method cannot fully decode ROIs coefficients before all BG coefficients are decoded because some residual sig- 
nificant bitplanes of ROIs is not shifted at the encoder. In this paper, a novel and flexible bitplanes shift coding method using bitplane classification is proposed, which can efficiently compress multiple ROIs with different degrees of interest and ensure all ROIs to be decoded before BG is decoded.

\section{PBAShift Method for Single ROI}

The PBAShift method is based on the facts that at low bit rates, ROIs in an image are desired to sustain higher quality than BG, while at the high bit rates, both ROI and BG can be coded with high quality and the difference between them is not very noticeable. So we divide the all bitplanes of ROIs and BG into three parts. For different significant parts, different shifting strategies are applied. Instead of shifting the bitplanes all at once by same scaling value $s$ as in Maxshift, the PBAShift method can code ROI in an image with two strategies-all bitplanes of the BG coefficients below all bitplanes of the ROI coefficients or all bitplanes of the BG coefficients below partial bitplanes of the ROI coefficients. In Figure 2, we compare the PSBhift with the new method.

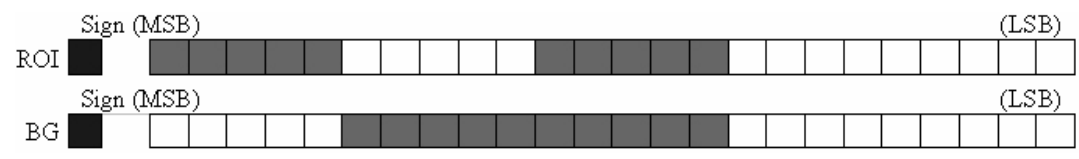

(a) PBAShift method

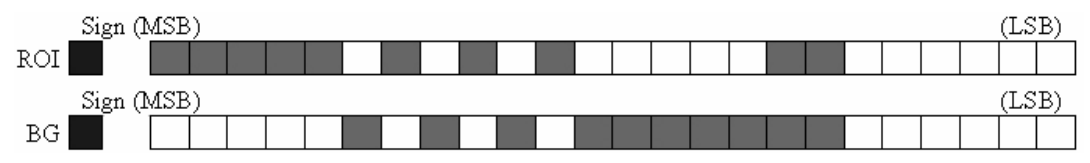

(b) PBAShift method

Fig. 2. Comparison of the PSBShift method and the PBAShift method for single ROI

We firstly define the parameters of PBAShift method as following,

1) $s_{1}$-The most significant bitplane number of the ROI coefficients.

2) $s_{2}$-The least significant bitplane number of the ROI coefficients.

3) $s_{3}$-The most significant bitplane number of the BG coefficients, which is also the general significant bitplane number of the ROI coefficients.

4) $s_{4}$-The least significant bitplane number of the BG coefficients.

In this paper, we index the bottom bitplane as bitplane 1, the next to bottom as bitplane 2, and so on. At the encoder, the bitplane shift scheme is as following, 
A) For any bitplane $b$ of an ROI coefficient,

a) If $b \leq s_{2}$, no shift and encoding directly.

b) If $s_{2}<b \leq s_{2}+s_{3}$, shift $b$ up to bitplane $s_{4}+2\left(b-s_{2}\right)-1$.

c) If $s_{2}+s_{3}<b \leq s_{1}+s_{2}+s_{3}$, shift $b$ up to bitplane $b+s_{4}+s_{3}-s_{2}$.

B) For any bitplane $b$ of an BG coefficient,

a) If $b \leq s_{4}$, no shift and encoding directly.

b) If $b>s_{4}$, shift $b$ up to bitplane $s_{4}+2\left(b-s_{4}\right)$.

At the decoder, for any given non-zero wavelet coefficient, the first step is to identify whether it is a bitplane of the ROI coefficient or the BG coefficient. The ROI decoding algorithm is presented as following,

A) If $b>s_{4}+2 s_{3}$, then $b \in R O I$, shift $b$ down to bitplane $b+s_{2}-s_{3}-s_{4}$.

B) If $b=s_{4}+2 i-1, i=1,2,3, \cdots, s_{3}$, Then $b \in R O I$, shift $b$ down to bitplane $\left(b-s_{4}+1\right) / 2+s_{2}$.

C) If $b=s_{4}+2 i, i=1,2,3, \cdots, s_{3}$, Then $b \in B G$, shift $b$ down to bitplane $\left(b-s_{4}\right) / 2+s_{4}$.

If the wavelet coefficient's MSB belongs to bitplanes of ROI, then it must be is an ROI coefficient. Otherwise, it is a BG coefficient. The bitplanes are then shifted back to their original levels by the decoding algorithm.

\section{PBAShift Method for Multiple ROIs}

In JPEG2000, both the Maxshift method and the general scaling based method can support the multiple ROI coding. However, each method has itself the drawbacks. The main drawback of Maxshift method is that the coefficient bitplanes of all ROIs must be scaled with the same values, which does not have the flexibility to allow for an arbitrary scaling value to define the relative importance of the ROIs and BG wavelet coefficients, and cannot code ROIs according to different degrees of interest. Additionally, in Maxshift method, all bitplanes of the BG coefficients cannot be decoded until the all bitplanes of all ROIs are decoded.

The general scaling based method can offer the multiple ROIs coding with different degrees of interest, but it has three major drawbacks. Firstly, it needs to encode the shape information of ROIs. This shape information significantly increases the complexity of encoder/decoder when the number of the ROIs increases. Secondly, when arbitrary ROI shapes are desired, the shape coding of the ROIs will consume a large number of bits, which reduces the overall coding efficiency. The current standard in JPEG2000 attempts to avoid this problem and only defines rectangle or ellipse shaped ROIs because they can be coded with a small number of bits. Finally, it is not 
convenient to deal with different wavelet subbands according to different degrees of interest, which is sometimes is very important to code and transmit for objectors.

In this paper, we propose a new and flexible multiple ROI coding methodPBAShift. The presented method not only can support arbitrary ROIs shape without shape coding, but also allows arbitrary scaling value between the ROIs and BG, which enables the flexible adjustment of compression quality in ROIs and BG according to different degrees of interest. The scheme of the PBAShift method for multiple ROI coding is illustrated in Fig. 3. The encoding and decoding method for multiple ROIs are similar to that for single ROI. However, three points must be noticed. Firstly, if $s_{2}$ of each ROI is different, we choose the minimum one to compute the shifting-back value of ROI bitplanes. Secondly, $s_{1}$ of each ROI must is equal. Thirdly, $s_{3}$ is equal to the most significant BG bitplane number, which is also equal to the maximum value of the general bitplane numbers of all ROIs. At low bit rates, different bitplanes are decoded with different degrees of ROI interest. At mediate bit rates, the most significant BG bitplanes and general significant ROI bitplanes can be decoded. At high bit rates, both ROIs and BG can be coded with high quality and difference between them is not very noticeable.

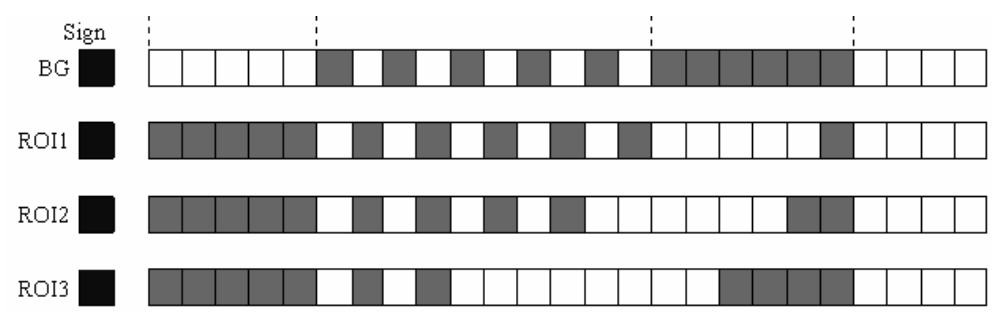

Fig. 3. The PBAShift method for multiple ROIs

\section{Experimental Results}

In Fig. 4, two reconstructed $512 \times 512$ remote sensing images-San Diego Shelter Island are given. They are coded with single ROI using PBAShift. In wavelet transform, we adopt $(5,3)$ integer wavelet filters. The original bitplane number is 8 . We define $s_{1}=4, s_{2}=1, s_{3}=3$, and $s_{4}=5$.

In Fig. 5, two figures give multiple ROI coding results for $512 \times 512$ Fishing Boat image at low bit rates and mediate bit rates. The people, the latter boat and the former boat are respectively defined as ROI-1, ROI-2 and ROI-3 in Fishing Boat image. The priority order of these ROIs is ROI-1>ROI-2>ROI-3. We hope that the ROI-1 has the best quality at low bit rates. The up-shifted numbers should be chosen as $\mathrm{s}_{\mathrm{ROI}-1}>\mathrm{S}_{\mathrm{ROI}}$ ${ }_{2}>\mathrm{S}_{\mathrm{ROI}-3}$, e.g., $s_{\mathrm{ROI}-1}=6, s_{\mathrm{ROI}-2}=5, s_{\mathrm{ROI}-3}=4$. The scaling value stored in the codestream is $s=\max \left(s_{R O I-1}, s_{R O I-2}, s_{R O I-3}\right)$. The reconstructed quality (PSNR) of three ROIs is shown in Fig. 6. From Fig. 5, it can be found that at low bit rates (e.g., bpp<1.0), all ROIs have the higher quality than BG. ROI-1 has the highest quality among three. 

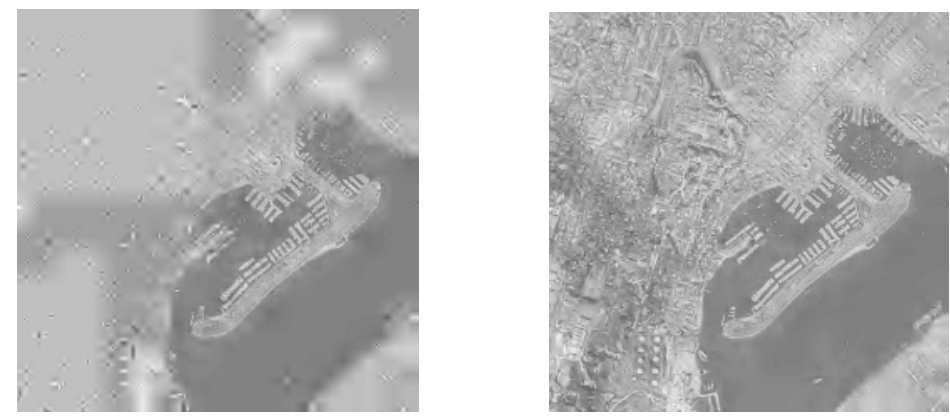

Fig. 4. The San Diego Shelter Island image with single ROI: 0.25 bpp (left), 1.0 bpp(right)

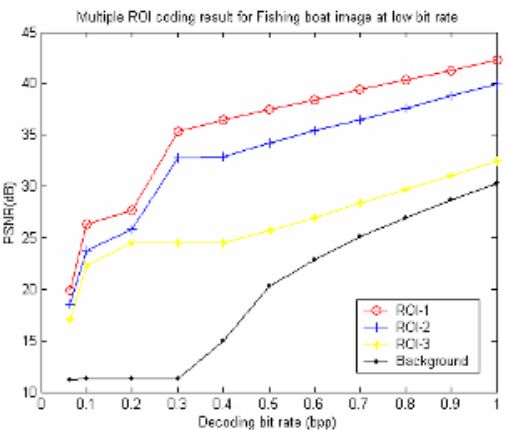

(a) Coding bit rates from 0.1 to $1.0 \mathrm{bpp}$

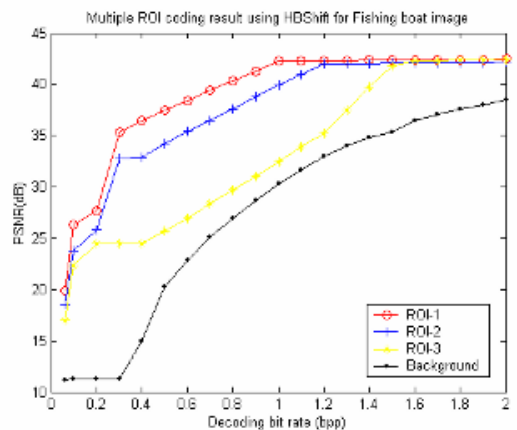

(b) Coding bit rates from 0.1 to $2.0 \mathrm{bpp}$

Fig. 5. Multiple ROI coding results for Fishing boat at low bit rates (a) and mediate bit rates (b)

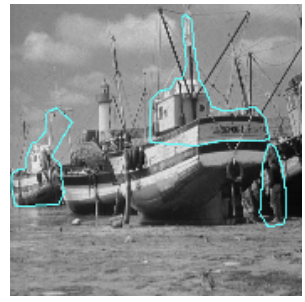

(a) Original image

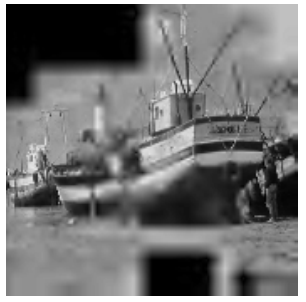

(b) 0.4 bpp

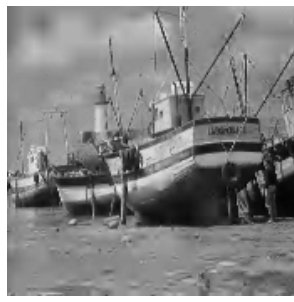

(c) $0.8 \mathrm{bpp}$

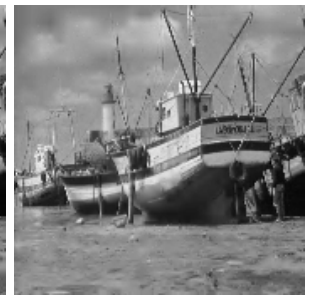

(d) $1.6 \mathrm{bpp}$

Fig. 6. The reconstructed Fishing boat image with three ROIs: ROI-1 is people, ROI-2 is the latter boat and ROI-3 is the former boat

ROIs. When the bit rates increases, the BG quality increases to some degree quickly. This is because the up-shifted numbers in the GSRB of ROI-2, ROI-3 are not large enough. Hence, the PBAShift method can support multiple ROI coding in a certain range of bit rates, which depends on the number of up-shifted bitplanes for each ROI. 


\section{Conclusions}

In this paper, a new ROI coding method so-called PBAShift is proposed. It has three primary advantages for ROI coding. Firstly, the new method can support arbitrary ROI shapes without coding shape information, which ensures the low complexity for coding ROIs in real-world applications. Secondly, the whole scaling values of all bitplanes are fewer than Maxshift method. Thirdly, the PBAShift can control flexibly the quality between the ROIs and BG by adjusting scaling values. Finally, the new method can support multiple ROI coding with different degrees of interest. We expect this idea is valuable for future research in ROI image coding and its applications.

\section{References}

1. ISO/IEC, ISO/IEC 15444-1, Information technology JPEG 2000 image coding system-Part 1: Core coding system. http://www.jpeg.org (2003).

2. ISO/IEC JTC 1/SC 29/WC 1 (ITU-Y SC8) JPEG 2000 Part Final Committee Draft Version 1.0, (2000), December.

3. A. Skodras, C. A. Christopoulos and T. Ebrahimi, The JPEG 2000 still image compression standard, Vol. 9, IEEE Signal Processing Magazine, (2001), 36-58.

4. C. Christopoulos, J. Askelf and M. Larsson, Efficient methods for encoding regions of interest in the upcoming JPEG 2000 still image coding standard, Vol. 7, IEEE Signal Processing Letters, (2000), (9), 247-249.

5. R. Grosbois, D. S. Cruz and T. Ebrahimi, New approach to JPEG 2000 compliant region of interest coding, Proc. Of the SPIE $46^{\text {th }}$ Annual Meeting, Applications of Digital Image Processing, San Diego, Vol. XXIV, CA, August, (2002).

6. Z. Wang and A. C. Bovik, Bitplane-by-Bitplane shift (BbBShift)-a suggestion for JPEG 2000 region of interest image coding, IEEE Signal Processing Letters, (2002), 9(5): 321324.

7. L. Liu and G. Fan, A new JPEG 2000 Region of interest image coding method: Partial Significant bitpanes shift, IEEE Signal Processing Letters, (2003), 10(4): 35-38.

8. Zhang Li-bao and Wang, Ke. Research on Regions Of Interest Coding Based on Compensation Scheme. Third International Symposium of Multispectral Image Processing and Pattern Recognition. Proc. SPIE. Vol. 5286. (2003), 931-934.

9. C. A. Christopoulos, A. Skodras and T. Ebrahimi, "The JPEG2000 still image coding system: An overview”, IEEE Transaction Consumer Electronics, (2000), 46(4):1103-1127 